Prepared in cooperation with the Indiana Office of Community and Rural Affairs

\title{
Vulnerable Transportation and Utility Assets near Actively Migrating Streams in Indiana
}

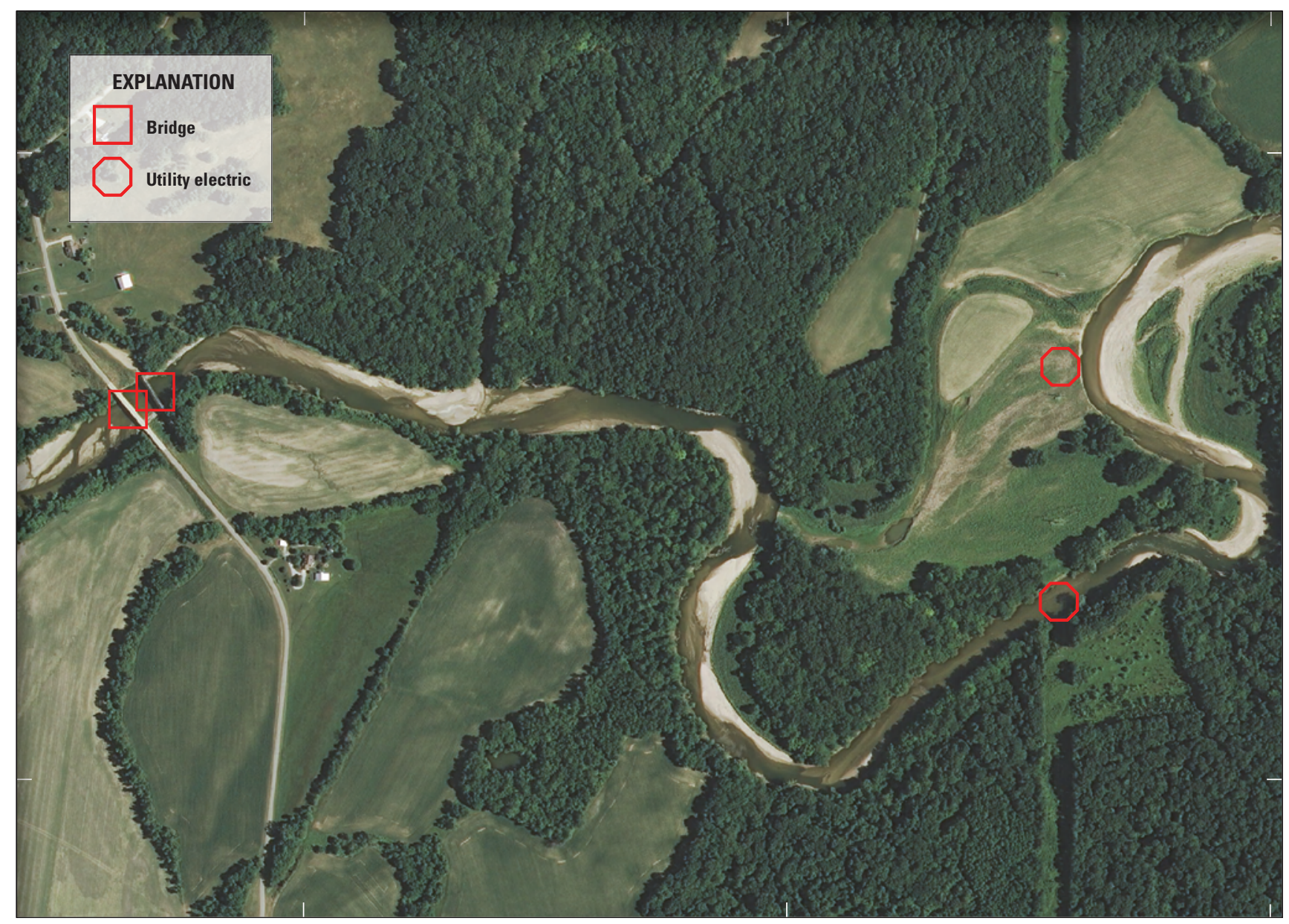

Data Series 1068 
Cover image. Digital orthophoto showing aerial perspective of vulnerable transportation and utility assets near an actively migrating reach of Sugar Creek in Parke County, Indiana. (Orthophotography from U.S. Department of Agriculture-FAS Aerial Photography Field Office, 2012.) 


\section{Vulnerable Transportation and Utility Assets near Actively Migrating Streams in Indiana}

By Benjamin J. Sperl

Prepared in cooperation with the Indiana Office of Community and Rural Affairs

Data Series 1068 


\title{
U.S. Department of the Interior \\ RYAN K. ZINKE, Secretary
}

\section{U.S. Geological Survey William H. Werkheiser, Acting Director}

\author{
U.S. Geological Survey, Reston, Virginia: 2017
}

For more information on the USGS - the Federal source for science about the Earth, its natural and living resources, natural hazards, and the environment-visit https://www.usgs.gov or call 1-888-ASK-USGS.

For an overview of USGS information products, including maps, imagery, and publications, visit https://store.usgs.gov.

Any use of trade, firm, or product names is for descriptive purposes only and does not imply endorsement by the U.S. Government.

Although this information product, for the most part, is in the public domain, it also may contain copyrighted materials as noted in the text. Permission to reproduce copyrighted items must be secured from the copyright owner.

Suggested citation:

Sperl, B.J., 2017, Vulnerable transportation and utility assets near actively migrating streams in Indiana: U.S. Geological Survey Data Series 1068, 11 p., https://doi.org/10.3133/ds1068.

ISSN 2327-636X (online) 


\section{Contents}

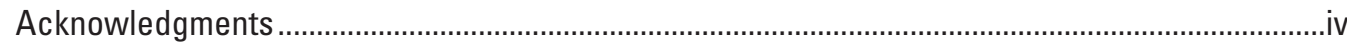

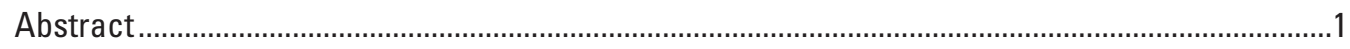

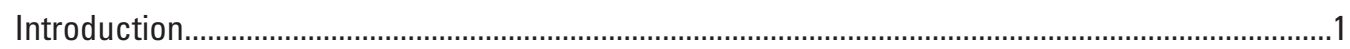

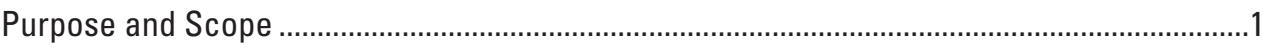

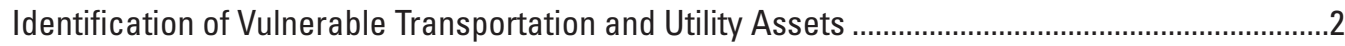

Polyline Shapefile of Investigated Stream Reaches..........................................................................10

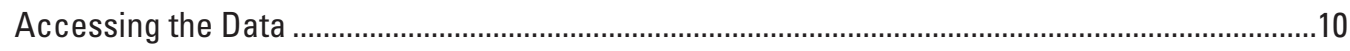

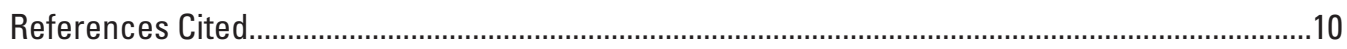

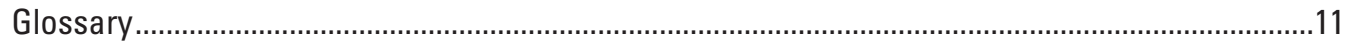

\section{Figures}

1. Map showing stream reaches identified as actively migrating or recently stationary..............................................................................................................

2. Digital orthophoto showing aerial perspective of vulnerable transportation and utility assets near an actively migrating reach of Sugar Creek in Parke County, Indiana

\section{Tables}

1. Sources and dates of imagery used to locate transportation and utility assets ...............2

2. Actively migrating stream reaches in Indiana, main stem and tributary ...........................4

3. Summary of vulnerable transportation and utility assets by stream reach, county, and class

4. Names and descriptions of attributes in Data_Series_2017_1068_

Channel_Migration_Rates.shp. 


\section{Conversion Factors}

U.S. customary units to International System of Units

\begin{tabular}{|c|c|c|}
\hline Multiply & By & To obtain \\
\hline \multicolumn{3}{|c|}{ Length } \\
\hline foot $(\mathrm{ft})$ & 0.3048 & meter $(\mathrm{m})$ \\
\hline mile (mi) & 1.609 & kilometer $(\mathrm{km})$ \\
\hline \multicolumn{3}{|c|}{ Migration rate } \\
\hline foot per year (ft/yr) & 0.3048 & meter per year (m/yr) \\
\hline
\end{tabular}

\section{Abbreviations}

FEH fluvial erosion hazard

GIS geographic information system

NHD National Hydrography Dataset

Words presented in bold type are defined in the Glossary section of this report.

\section{Acknowledgments}

The author acknowledges the support of the Indiana Office of Community and Rural Affairs and members of the Indiana Silver Jackets, particularly Bret Robinson and Robert Barr, for their contributions to the fluvial erosion hazards program in Indiana. Work performed by fluvial geomorphologist Bret Robinson (retired) of the U.S. Geological Survey provided original data released with this report. His efforts are greatly appreciated. 


\title{
Vulnerable Transportation and Utility Assets near Actively Migrating Streams in Indiana
}

\author{
By Benjamin J. Sperl
}

\begin{abstract}
An investigation was completed by the U.S. Geological Survey in cooperation with the Indiana Office of Community and Rural Affairs that found 1,132 transportation and utility assets in Indiana are vulnerable to fluvial erosion hazards due to close proximity to actively migrating streams. Locations of transportation assets (bridges, roadways, and railroad lines) and selected utility assets (high-capacity overhead powertransmission lines, underground pipelines, water treatment facilities, and in-channel dams) were determined using aerial imagery hosted by the Google Earth platform. Identified assets were aggregated by stream reach, county, and class. Accompanying the report is a polyline shapefile of the stream reaches documented by Robinson (2013a). The shapefile, derived from line work in the National Hydrography Dataset and attributed with channel migration rates, is released with complete Federal Geographic Data Committee metadata. The data presented in this report are intended to help stakeholders and others identify high-risk areas where transportation and utility assets may be threatened by fluvial erosion hazards thus warranting consideration for mitigation strategies.
\end{abstract}

\section{Introduction}

The June 7-9, 2008, flooding in central and southern Indiana caused extensive erosion-related damages to public and private infrastructure (Morlock and others, 2008).

Following the flood, members of the Indiana Silver Jackets (https://silverjackets.nfrmp.us/State-Teams/Indiana), a multiagency taskforce whose mission is to mitigate natural hazards in Indiana, recognized the need for a program that focuses specifically on fluvial erosion hazards (FEH). FEH is a term that collectively refers to "damages imparted by erosion and stream-channel migration processes" (Robinson, 2013b, p. 1).
The FEH program (http://feh.iupui.edu/) was formed to help communities understand and reduce the risks associated with fluvial erosion. The program meets that goal through education and outreach as well as sponsorship of projects that produce tools and information to enhance awareness of the hazard (Indiana University-Purdue University Indianapolis, 2017). Projects sponsored by the FEH program have developed regional curves for bankfull-channel dimension for Indiana streams, measured the rates of channel migration, and delineated FEH-avoidance corridors along actively migrating stream reaches (Robinson, 2013a; Robinson, 2013b; Indiana Department of Natural Resources, 2016).

Rates of channel migration were measured along 42 stream reaches in Indiana between 1998 and 2011 (Robinson, 2013a). Of the 42 stream reaches examined, 16 were found to be actively migrating with a migration rate of greater than 1 foot per year, while 26 were found to be stationary, which is defined as having a migration rate of less than 1 foot per year, over the period of study (Robinson, 2013a).

\section{Purpose and Scope}

The purpose of this report is (1) to publish a table highlighting stream reaches and counties in Indiana where transportation and utility assets are at greater risk for damages by fluvial erosion based on the findings of Robinson (2013a) and (2) to document a geographic information system (GIS) dataset (Sperl, 2017) of those stream reaches which may serve as a resource to stakeholders and others in conducting their own independent risk analyses. The report focuses solely on transportation and utility assets, including bridges, roadways, railroad lines, high-capacity overhead power-transmission lines, underground pipelines, water treatment facilities, and in-channel dams. 


\section{Identification of Vulnerable Transportation and Utility Assets}

The locations of vulnerable assets were determined visually using digital orthophotography hosted by the Google Earth platform. The availability of imagery provided in Google Earth varies by geographic area and date; therefore, assets were identified from multiple images covering different dates and areal swaths. When measuring distances between assets and eroding channel banks, an effort was made to use the most currently available imagery with respect to the time of the investigation in 2014. However, in many cases, images with older capture dates were used instead of the most currently available imagery because they offered finer ground resolution or leaf-off conditions making assets more visible. The sources and dates of imagery used to identify vulnerable assets are summarized in table 1 . The identified assets are near or within the fluvial plains of the 16 active stream reaches documented in Robinson (2013a), as well as 38 tributaries where migration rates were not directly measured but also show signs of recent channel migration (fig. 1; table 2).

To determine the degree of channel migration in tributaries, digital orthophotos from different capture dates were used to compare the position of stream channels relative to fixed landscape features. Through this method, many tributary channels were identified as static. However, 38 tributaries showed signs of observable channel movement in the time span between the capture dates of digital orthophotos. These 38 tributaries were noted as actively migrating but no measurements were completed to document their channel-migration rates as they were for main stem stream reaches in Robinson (2013a).

Tools provided in Google Earth were used to record the locations of assets and store their geographic coordinates in a placemark file (.kmz). Beginning at the most downstream point on a stream reach and panning over imagery in the upstream direction, locations of assets were recorded where their position relative to the banks of an actively migrating stream was considered a potential vulnerability. As a general rule, an asset was judged vulnerable if the shortest distance from its location to the nearest channel bank was approximately 300 feet or less. The locations of some buried pipelines that were not visible from imagery were identified with the aid of a GIS dataset containing polyline features that represent the pipes' underground pathways
Table 1. Sources and dates of imagery used to locate transportation and utility assets.

[Date format mm/dd/yyyy, month/day/year; USDA, U.S. Department of Agriculture]

\begin{tabular}{lcc}
\hline \multicolumn{1}{c}{ Image source $^{1}$} & Image date & $\begin{array}{c}\text { Number of } \\
\text { assets }\end{array}$ \\
\hline Google & $10 / 10 / 2013$ & 3 \\
\hline Google & $10 / 11 / 2013$ & 15 \\
\hline Google & $2 / 26 / 2012$ & 19 \\
\hline Google & $4 / 4 / 2013$ & 36 \\
\hline Google & $5 / 14 / 2012$ & 10 \\
\hline Google & $5 / 5 / 2010$ & 21 \\
\hline Google & $8 / 29 / 2012$ & 36 \\
\hline Google & $9 / 1 / 2011$ & 67 \\
\hline Google & $9 / 13 / 2013$ & 5 \\
\hline Google & $9 / 22 / 2013$ & 142 \\
\hline Google & $9 / 25 / 2013$ & 34 \\
\hline Indiana Map Framework Data & $2 / 28 / 2005$ & 703 \\
\hline USDA Farm Service Agency & $6 / 18 / 2008$ & 1 \\
\hline USDA Farm Service Agency & $6 / 19 / 2010$ & 25 \\
\hline USDA Farm Service Agency & $9 / 14 / 2011$ & 15 \\
\hline
\end{tabular}

${ }^{1}$ Refers to ownership of imagery; sensor specifications are not provided.

${ }^{2}$ Date image was captured.

(Indiana Geological Survey, 2002). In some cases, the locations of pipelines could be inferred from vegetation clearings along utility easements, like the one shown for an electric utility in figure 2. Stream reaches that remained stationary over the period of study in Robinson (2013a) were excluded from the investigation for vulnerable assets, but they are included as features in the polyline shapefile (Sperl, 2017).

As locations of assets were recorded, so too were the following attributes: name of the nearby stream reach and its main stem stream reach, county where the asset was found, and the class of an asset (bridge, road, electric utility, etc.). These attributes were used to aggregate the data and summarize the results by stream reach, county, and class in table 3 . 


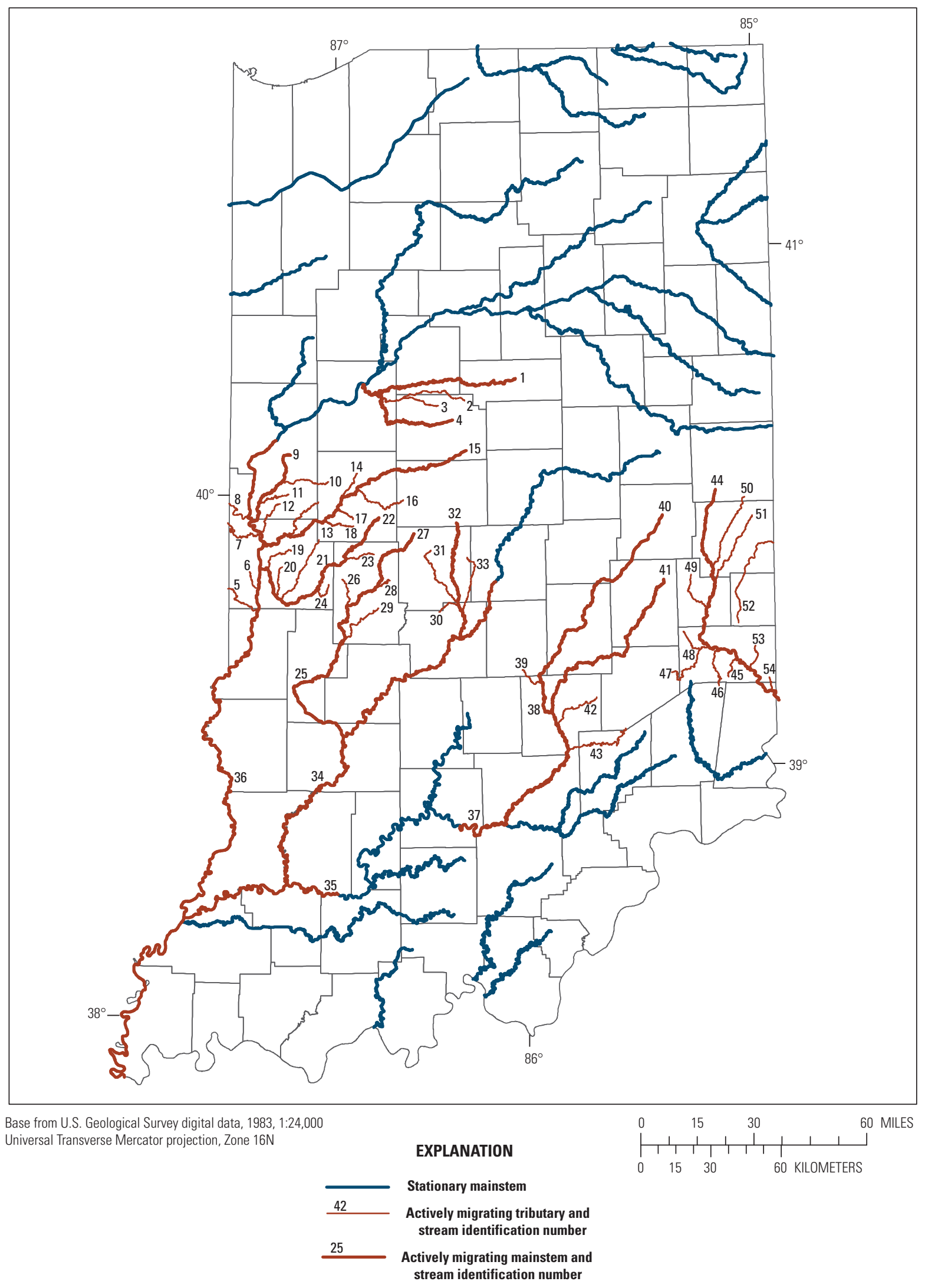

Figure 1. Map showing stream reaches identified as actively migrating or recently stationary. (Map labels 1-54 reference table 2). 
Table 2. Actively migrating stream reaches in Indiana, main stem and tributary.

[mi, miles; ft/yr, feet per year; --, no data]

\begin{tabular}{|c|c|c|c|c|}
\hline $\begin{array}{l}\text { Map label } \\
\text { (fig. 1) }^{1}\end{array}$ & Reach name & Main stem & Length (mi) & $\begin{array}{c}\text { Migration rate } \\
(\mathrm{ft} / \mathrm{yr})^{2}\end{array}$ \\
\hline 1 & Wildcat Creek & Wildcat Creek & 68.9 & 5.1 \\
\hline 2 & Middle Fork Wildcat Creek & South Fork Wildcat Creek & 33.8 & -- \\
\hline 3 & Campbells Run & South Fork Wildcat Creek & 13.2 & -- \\
\hline 4 & South Fork Wildcat Creek & South Fork Wildcat Creek & 44.9 & 8.0 \\
\hline 5 & Brouilletts Creek & Wabash River (lower) & 15.4 & -- \\
\hline 6 & Norton Creek & Wabash River (lower) & 6.7 & -- \\
\hline 7 & Little Vermilion River & Wabash River (lower) & 21.4 & -- \\
\hline 8 & Vermilion River & Wabash River (lower) & 10.9 & -- \\
\hline 9 & Coal Creek & Coal Creek & 32.7 & 4.5 \\
\hline 10 & East Fork Coal Creek & Coal Creek & 17.1 & -- \\
\hline 11 & Prairie Creek & Coal Creek & 13.1 & -- \\
\hline 12 & Mill Creek & Wabash River (lower) & 13.4 & -- \\
\hline 13 & Sugar Mill Creek & Sugar Creek & 20.8 & -- \\
\hline 14 & Black Creek & Sugar Creek & 9.7 & -- \\
\hline 15 & Sugar Creek & Sugar Creek & 87.7 & 6.4 \\
\hline 16 & Walnut Fork Sugar Creek & Sugar Creek & 23.3 & -- \\
\hline 17 & Rattlesnake Creek & Sugar Creek & 8.4 & -- \\
\hline 18 & Indian Creek & Sugar Creek & 13.6 & -- \\
\hline 19 & Leatherwood Creek & Big Raccoon Creek & 12.5 & -- \\
\hline 20 & Rock Run & Big Raccoon Creek & 10.5 & -- \\
\hline 21 & Little Raccoon Creek & Big Raccoon Creek & 23.2 & -- \\
\hline 22 & Big Raccoon Creek & Big Raccoon Creek & 68.3 & 6.3 \\
\hline 23 & Ramp Creek & Big Raccoon Creek & 10.3 & -- \\
\hline 24 & Rocky Fork & Big Raccoon Creek & 8.0 & -- \\
\hline 25 & Eel River (south) & Eel River (south) & 54.9 & 5.4 \\
\hline 26 & Little Walnut Creek & Big Walnut Creek & 9.5 & -- \\
\hline 27 & Big Walnut Creek & Big Walnut Creek & 54.2 & 12.2 \\
\hline 28 & Clear Creek & Big Walnut Creek & 6.8 & -- \\
\hline 29 & Deer Creek & Eel River (south) & 18.5 & -- \\
\hline 30 & McCracken Creek & White Lick Creek & 8.3 & -- \\
\hline 31 & West Fork White Lick Creek & White Lick Creek & 25.3 & -- \\
\hline 32 & White Lick Creek & White Lick Creek & 45.8 & 16.3 \\
\hline 33 & East Fork White Lick Creek & White Lick Creek & 20.8 & -- \\
\hline 34 & White River (lower) & White River (lower) & 229.0 & 16.5 \\
\hline 35 & East Fork White River (lower) & East Fork White River (lower) & 21.7 & 8.0 \\
\hline 36 & Wabash River (lower) & Wabash River (lower) & 275.9 & 8.3 \\
\hline 37 & East Fork White River (upper) & East Fork White River (upper) & 86.9 & 12.3 \\
\hline 38 & Driftwood River & Driftwood River & 15.8 & 5.5 \\
\hline 39 & Nineveh Creek & Driftwood River & 9.1 & -- \\
\hline 40 & Big Blue River & Big Blue River & 74.1 & 4.1 \\
\hline 41 & Flatrock River & Flatrock River & 74.4 & 10.2 \\
\hline 42 & Clifty Creek & East Fork White River (upper) & 19.1 & -- \\
\hline 43 & Sand Creek & East Fork White River (upper) & 31.1 & \\
\hline
\end{tabular}


Table 2. Actively migrating stream reaches in Indiana, main stem and tributary.-Continued

[mi, miles; ft/yr, feet per year; --, no data]

\begin{tabular}{|c|c|c|c|c|}
\hline $\begin{array}{c}\text { Map label } \\
\text { (fig. 1) }^{1}\end{array}$ & Reach name & Main stem & Length (mi) & $\begin{array}{c}\text { Migration rate } \\
(\mathrm{ft} / \mathrm{yr})^{2}\end{array}$ \\
\hline 44 & Whitewater River & Whitewater River & 93.1 & 30.2 \\
\hline 45 & Blue Creek & Whitewater River & 7.7 & -- \\
\hline 46 & Pipe Creek & Whitewater River & 15.3 & -- \\
\hline 47 & Salt Creek & Whitewater River & 23.6 & -- \\
\hline 48 & Little Salt Creek & Whitewater River & 9.5 & -- \\
\hline 49 & Williams Creek & Whitewater River & 15.0 & -- \\
\hline 50 & Greens Fork & Whitewater River & 26.5 & -- \\
\hline 51 & Nolands Fork & Whitewater River & 26.1 & -- \\
\hline 52 & East Fork Whitewater River & Whitewater River & 31.7 & -- \\
\hline 53 & Big Cedar Creek & Whitewater River & 10.1 & -- \\
\hline 54 & Johnson Fork & Whitewater River & 4.5 & -- \\
\hline
\end{tabular}

${ }^{1}$ Map labels reference figure 1.

${ }^{2}$ Channel migration rates were measured in a report by Robinson (2013a) for mainstem stream reaches only.

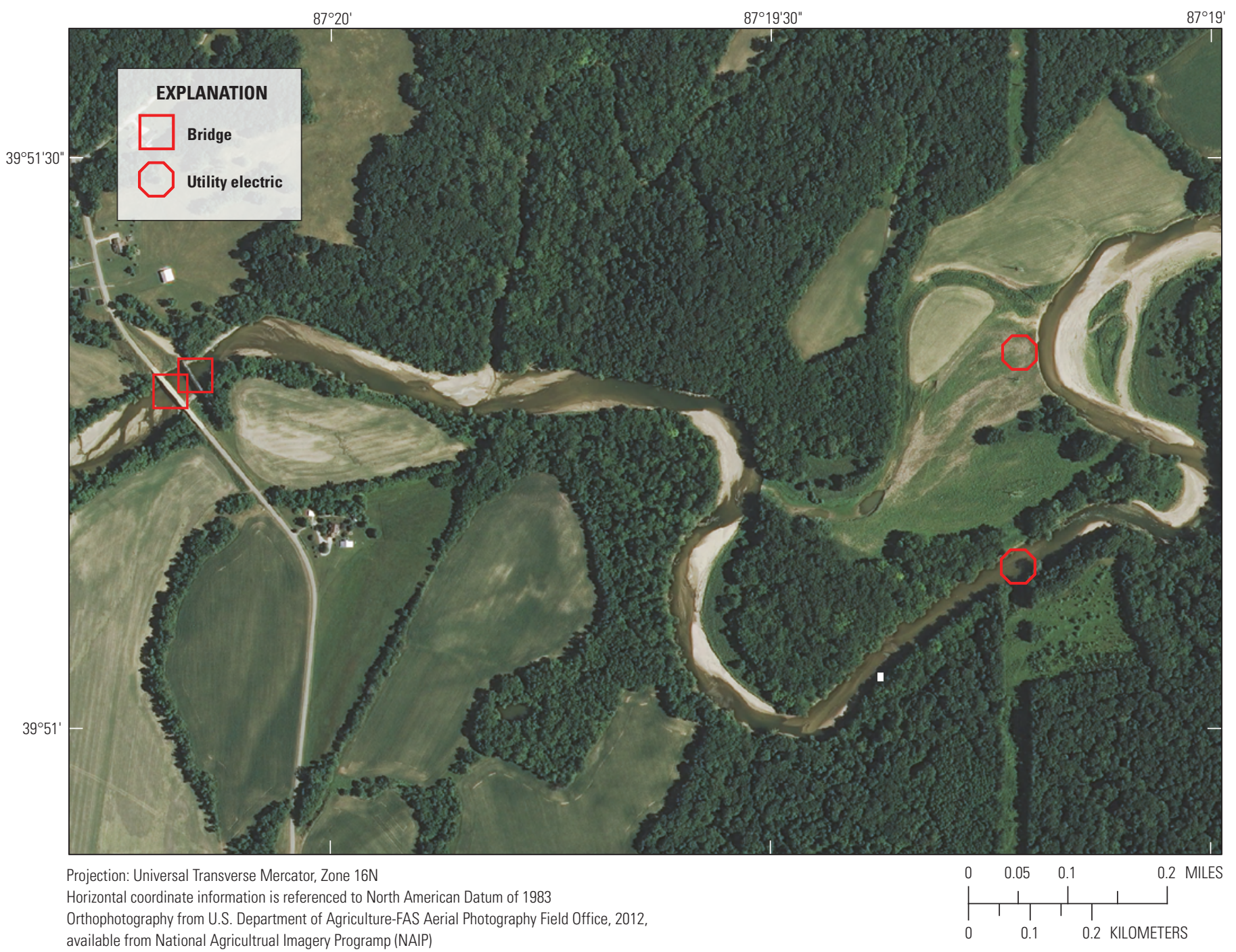

Figure 2. Digital orthophoto showing aerial perspective of vulnerable transportation and utility assets near an actively migrating reach of Sugar Creek in Parke County, Indiana. 
Table 3. Summary of vulnerable transportation and utility assets by stream reach, county, and class.

[Values represent the number of vulnerable assets found, aggregated by stream reach, county, and class; --, no data]

\begin{tabular}{|c|c|c|c|c|c|c|c|c|c|c|c|}
\hline \multirow[b]{2}{*}{ Reach name } & \multirow[b]{2}{*}{ Main stem } & \multicolumn{4}{|c|}{ Transportation } & \multicolumn{6}{|c|}{ Utility } \\
\hline & & Road & Bridge & Railroad & $\begin{array}{c}\text { Railroad } \\
\text { bridge }\end{array}$ & Dam & Electric & Levee & 0il/gas & Water & Total \\
\hline \multicolumn{12}{|c|}{ Bartholomew County } \\
\hline Driftwood River & Driftwood River & 2 & 5 & -- & -- & -- & 1 & -- & 3 & -- & 11 \\
\hline Nineveh Creek & Driftwood River & -- & 4 & -- & -- & -- & -- & -- & -- & -- & 4 \\
\hline Clifty Creek & East Fork White River (upper) & -- & -- & -- & -- & -- & -- & -- & 2 & -- & 2 \\
\hline East Fork White River (upper) & East Fork White River (upper) & 1 & 2 & -- & 1 & 1 & 3 & -- & 1 & -- & 9 \\
\hline Flatrock River & Flatrock River & 3 & 8 & 1 & 1 & 1 & 3 & -- & 3 & -- & 20 \\
\hline \multicolumn{12}{|c|}{ Carroll County } \\
\hline Wildcat Creek & Wildcat Creek & -- & 2 & -- & -- & -- & -- & -- & -- & -- & 2 \\
\hline \multicolumn{12}{|c|}{ Clay County } \\
\hline Eel River (south) & Eel River (south) & 16 & 8 & -- & -- & -- & 2 & -- & 2 & -- & 28 \\
\hline \multicolumn{12}{|c|}{ Clinton County } \\
\hline Campbells Run & South Fork Wildcat Creek & -- & 4 & -- & -- & -- & -- & -- & -- & -- & 4 \\
\hline South Fork Wildcat Creek & South Fork Wildcat Creek & 5 & 6 & 1 & 1 & -- & -- & -- & -- & -- & 13 \\
\hline \multicolumn{12}{|c|}{ Daviess County } \\
\hline East Fork White River (lower) & East Fork White River (lower) & -- & 2 & -- & 1 & -- & 3 & -- & 1 & -- & 7 \\
\hline White River (lower) & White River (lower) & -- & -- & 3 & 2 & -- & -- & -- & -- & -- & 5 \\
\hline \multicolumn{12}{|c|}{ Dearborn County } \\
\hline Whitewater River & Whitewater River & 12 & 4 & 3 & -- & 1 & -- & -- & -- & -- & 20 \\
\hline \multicolumn{12}{|c|}{ Fayette County } \\
\hline Nolands Fork & Whitewater River & -- & 1 & -- & -- & -- & -- & -- & -- & -- & 1 \\
\hline Whitewater River & Whitewater River & 4 & 4 & 1 & 2 & -- & 3 & -- & -- & -- & 14 \\
\hline Williams Creek & Whitewater River & 3 & 2 & -- & -- & -- & -- & -- & -- & -- & 5 \\
\hline \multicolumn{12}{|c|}{ Fountain County } \\
\hline Coal Creek & Coal Creek & 11 & 10 & -- & 1 & 1 & 1 & -- & -- & -- & 24 \\
\hline Prairie Creek & Coal Creek & 3 & 7 & -- & -- & -- & 3 & -- & -- & -- & 13 \\
\hline Wabash River (lower) & Wabash River (lower) & 1 & 2 & -- & 1 & -- & -- & -- & -- & -- & 4 \\
\hline \multicolumn{12}{|c|}{ Franklin County } \\
\hline Big Cedar Creek & Whitewater River & 6 & 3 & -- & 1 & -- & 1 & -- & -- & -- & 11 \\
\hline Little Salt Creek & Whitewater River & -- & 1 & -- & -- & -- & 1 & -- & -- & -- & 2 \\
\hline Pipe Creek & Whitewater River & 6 & 4 & -- & -- & -- & -- & -- & -- & -- & 10 \\
\hline
\end{tabular}


Table 3. Summary of vulnerable transportation and utility assets by stream reach, county, and class. - Continued

[Values represent the number of vulnerable assets found, aggregated by stream reach, county, and class; --, no data]

\begin{tabular}{|c|c|c|c|c|c|c|c|c|c|c|c|}
\hline \multirow[b]{2}{*}{ Reach name } & \multirow[b]{2}{*}{ Main stem } & \multicolumn{4}{|c|}{ Transportation } & \multicolumn{6}{|c|}{ Utility } \\
\hline & & Road & Bridge & Railroad & $\begin{array}{c}\text { Railroad } \\
\text { bridge }\end{array}$ & Dam & Electric & Levee & Oil/gas & Water & Total \\
\hline \multicolumn{12}{|c|}{ Franklin County-Continued } \\
\hline Salt Creek & Whitewater River & 4 & 2 & -- & -- & -- & 1 & -- & -- & -- & 7 \\
\hline Whitewater River & Whitewater River & 32 & 7 & 19 & 1 & 1 & 3 & -- & 4 & -- & 67 \\
\hline \multicolumn{12}{|c|}{ Gibson County } \\
\hline Wabash River (lower) & Wabash River (lower) & -- & 1 & -- & 3 & -- & -- & -- & 9 & -- & 13 \\
\hline White River (lower) & White River (lower) & 1 & -- & -- & -- & -- & -- & -- & -- & -- & 1 \\
\hline \multicolumn{12}{|c|}{ Greene County } \\
\hline Eel River (south) & Eel River (south) & -- & 1 & 1 & -- & -- & 1 & -- & 3 & -- & 6 \\
\hline White River (lower) & White River (lower) & 3 & 3 & 1 & 3 & -- & 7 & -- & 2 & -- & 19 \\
\hline \multicolumn{12}{|c|}{ Hancock County } \\
\hline Big Blue River & Big Blue River & 1 & 3 & -- & -- & -- & 1 & -- & -- & -- & 5 \\
\hline \multicolumn{12}{|c|}{ Hendricks County } \\
\hline Big Walnut Creek & Big Walnut Creek & -- & 4 & -- & -- & -- & -- & -- & -- & -- & 4 \\
\hline East Fork White Lick Creek & White Lick Creek & 3 & 3 & -- & -- & -- & -- & -- & -- & -- & 6 \\
\hline West Fork White Lick Creek & White Lick Creek & 6 & 21 & -- & 1 & -- & 2 & -- & -- & 1 & 31 \\
\hline White Lick Creek & White Lick Creek & 16 & 15 & -- & 1 & -- & 9 & -- & 2 & 1 & 44 \\
\hline \multicolumn{12}{|c|}{ Henry County } \\
\hline Big Blue River & Big Blue River & 1 & 8 & -- & -- & -- & -- & -- & -- & -- & 9 \\
\hline \multicolumn{12}{|c|}{ Jackson County } \\
\hline East Fork White River (upper) & East Fork White River (upper) & 8 & 16 & 6 & 2 & 1 & 3 & -- & 2 & 1 & 39 \\
\hline Sand Creek & East Fork White River (upper) & -- & 2 & -- & -- & -- & -- & -- & -- & -- & 2 \\
\hline \multicolumn{12}{|c|}{ Jennings County } \\
\hline Sand Creek & East Fork White River (upper) & 1 & -- & -- & -- & -- & -- & -- & 2 & -- & 3 \\
\hline \multicolumn{12}{|c|}{ Johnson County } \\
\hline Big Blue River & Big Blue River & -- & 4 & -- & 1 & 1 & 3 & -- & 4 & 1 & 14 \\
\hline \multicolumn{12}{|c|}{ Knox County } \\
\hline Wabash River (lower) & Wabash River (lower) & -- & 4 & -- & 1 & -- & -- & 1 & 9 & -- & 15 \\
\hline White River (lower) & White River (lower) & 6 & 8 & -- & 2 & -- & 7 & 3 & 14 & 1 & 41 \\
\hline \multicolumn{12}{|c|}{ Lawrence County } \\
\hline East Fork White River (upper) & East Fork White River (upper) & 6 & 2 & 2 & -- & -- & 1 & -- & 3 & -- & 14 \\
\hline
\end{tabular}


Table 3. Summary of vulnerable transportation and utility assets by stream reach, county, and class. - Continued

[Values represent the number of vulnerable assets found, aggregated by stream reach, county, and class; --, no data]

\begin{tabular}{|c|c|c|c|c|c|c|c|c|c|c|c|}
\hline \multirow[b]{2}{*}{ Reach name } & \multirow[b]{2}{*}{ Main stem } & \multicolumn{4}{|c|}{ Transportation } & \multicolumn{6}{|c|}{ Utility } \\
\hline & & Road & Bridge & Railroad & $\begin{array}{c}\text { Railroad } \\
\text { bridge }\end{array}$ & Dam & Electric & Levee & Oil/gas & Water & Total \\
\hline \multicolumn{12}{|c|}{ Marion County } \\
\hline White River (lower) & White River (lower) & -- & 1 & -- & -- & -- & 1 & -- & 2 & -- & 4 \\
\hline \multicolumn{12}{|c|}{ Montgomery County } \\
\hline Black Creek & Sugar Creek & 3 & 3 & -- & -- & -- & -- & -- & -- & -- & 6 \\
\hline Indian Creek & Sugar Creek & -- & -- & -- & -- & -- & -- & -- & 1 & -- & 1 \\
\hline Rattlesnake Creek & Sugar Creek & 1 & 1 & -- & -- & -- & -- & -- & -- & -- & 2 \\
\hline Sugar Creek & Sugar Creek & 7 & 15 & 1 & 1 & -- & -- & -- & -- & -- & 24 \\
\hline \multicolumn{12}{|c|}{ Morgan County } \\
\hline East Fork White Lick Creek & White Lick Creek & 4 & 6 & -- & 1 & -- & -- & -- & 1 & 1 & 13 \\
\hline McCracken Creek & White Lick Creek & -- & 2 & -- & -- & -- & -- & -- & -- & -- & 2 \\
\hline White Lick Creek & White Lick Creek & 4 & 7 & -- & 1 & -- & 7 & -- & 3 & -- & 22 \\
\hline White River (lower) & White River (lower) & 6 & 5 & 1 & 2 & 1 & 10 & -- & 6 & -- & 31 \\
\hline \multicolumn{12}{|c|}{ Owen County } \\
\hline Eel River (south) & Eel River (south) & 1 & 2 & -- & -- & -- & -- & -- & -- & -- & 3 \\
\hline White River (lower) & White River (lower) & 6 & 5 & 9 & -- & -- & 3 & -- & 1 & -- & 24 \\
\hline \multicolumn{12}{|c|}{ Parke County } \\
\hline Big Raccoon Creek & Big Raccoon Creek & 11 & 17 & -- & -- & 3 & 3 & -- & 6 & -- & 40 \\
\hline Leatherwood Creek & Big Raccoon Creek & 1 & 4 & -- & -- & -- & 1 & -- & -- & -- & 6 \\
\hline Little Raccoon Creek & Big Raccoon Creek & -- & 7 & -- & -- & -- & -- & -- & -- & -- & 7 \\
\hline Rock Run & Big Raccoon Creek & -- & 2 & -- & -- & -- & 1 & -- & -- & -- & 3 \\
\hline Rocky Fork & Big Raccoon Creek & 1 & 4 & -- & -- & -- & -- & -- & -- & -- & 5 \\
\hline Coal Creek & Coal Creek & -- & 1 & -- & -- & -- & -- & -- & -- & -- & 1 \\
\hline Sugar Creek & Sugar Creek & 6 & 9 & -- & -- & 1 & 10 & -- & 2 & -- & 28 \\
\hline Sugar Mill Creek & Sugar Creek & 2 & 4 & -- & -- & -- & -- & -- & -- & -- & 6 \\
\hline Mill Creek & Wabash River (lower) & 2 & 4 & -- & -- & -- & -- & -- & -- & -- & 6 \\
\hline Wabash River (lower) & Wabash River (lower) & -- & 3 & -- & 2 & -- & 2 & -- & 5 & -- & 12 \\
\hline \multicolumn{12}{|c|}{ Pike County } \\
\hline White River (lower) & White River (lower) & -- & -- & 1 & -- & -- & -- & -- & -- & -- & 1 \\
\hline \multicolumn{12}{|c|}{ Posey County } \\
\hline Wabash River (lower) & Wabash River (lower) & -- & 4 & -- & 1 & -- & -- & -- & 9 & -- & 14 \\
\hline
\end{tabular}


Table 3. Summary of vulnerable transportation and utility assets by stream reach, county, and class. - Continued

[Values represent the number of vulnerable assets found, aggregated by stream reach, county, and class; --, no data]

\begin{tabular}{|c|c|c|c|c|c|c|c|c|c|c|c|}
\hline \multirow[b]{2}{*}{ Reach name } & \multirow[b]{2}{*}{ Main stem } & \multicolumn{4}{|c|}{ Transportation } & \multicolumn{6}{|c|}{ Utility } \\
\hline & & Road & Bridge & Railroad & $\begin{array}{c}\text { Railroad } \\
\text { bridge }\end{array}$ & Dam & Electric & Levee & 0il/gas & Water & Total \\
\hline \multicolumn{12}{|c|}{ Putnam County } \\
\hline Big Raccoon Creek & Big Raccoon Creek & 5 & 4 & -- & -- & -- & 1 & -- & 1 & -- & 11 \\
\hline Ramp Creek & Big Raccoon Creek & 3 & 5 & -- & -- & -- & & -- & 2 & -- & 10 \\
\hline Big Walnut Creek & Big Walnut Creek & 26 & 27 & -- & 3 & 1 & 3 & -- & 8 & -- & 68 \\
\hline Clear Creek & Big Walnut Creek & 2 & 4 & -- & -- & -- & -- & -- & -- & -- & 6 \\
\hline Little Walnut Creek & Big Walnut Creek & 12 & 4 & -- & 1 & -- & -- & -- & -- & -- & 17 \\
\hline Deer Creek & Eel River (south) & -- & 2 & -- & -- & -- & -- & -- & -- & -- & 2 \\
\hline Eel River (south) & Eel River (south) & -- & -- & -- & -- & -- & 1 & -- & -- & -- & 1 \\
\hline \multicolumn{12}{|c|}{ Rush County } \\
\hline Big Blue River & Big Blue River & 6 & 4 & -- & 1 & -- & -- & -- & -- & -- & 11 \\
\hline \multicolumn{12}{|c|}{ Shelby County } \\
\hline Big Blue River & Big Blue River & 17 & 12 & -- & 2 & -- & 14 & -- & 4 & 1 & 50 \\
\hline Flatrock River & Flatrock River & 4 & 3 & -- & -- & -- & -- & -- & -- & -- & 7 \\
\hline \multicolumn{12}{|c|}{ Sullivan County } \\
\hline Wabash River (lower) & Wabash River (lower) & 2 & 1 & -- & 1 & -- & 1 & - & 4 & - & 9 \\
\hline \multicolumn{12}{|c|}{ Tippecanoe County } \\
\hline Middle Fork Wildcat Creek & South Fork Wildcat Creek & 2 & 5 & -- & -- & -- & 1 & -- & 1 & -- & 9 \\
\hline South Fork Wildcat Creek & South Fork Wildcat Creek & 7 & 7 & -- & 1 & -- & 4 & -- & 5 & -- & 24 \\
\hline Wildcat Creek & Wildcat Creek & 8 & 7 & -- & 1 & -- & 4 & -- & 3 & -- & 23 \\
\hline \multicolumn{12}{|c|}{ Union County } \\
\hline East Fork Whitewater River & Whitewater River & 3 & 3 & -- & 1 & -- & -- & -- & -- & -- & 7 \\
\hline \multicolumn{12}{|c|}{ Vermillion County } \\
\hline Little Vermilion River & Wabash River (lower) & 2 & 7 & -- & 1 & -- & 1 & -- & -- & -- & 11 \\
\hline Vermilion River & Wabash River (lower) & 3 & 5 & -- & -- & -- & 1 & -- & -- & -- & 9 \\
\hline Wabash River (lower) & Wabash River (lower) & -- & 3 & 1 & -- & -- & 1 & -- & -- & -- & 5 \\
\hline \multicolumn{12}{|c|}{ Vigo County } \\
\hline Brouilletts Creek & Wabash River (lower) & -- & -- & -- & -- & -- & -- & -- & 2 & -- & 2 \\
\hline Wabash River (lower) & Wabash River (lower) & 4 & 6 & -- & 3 & -- & 10 & -- & 9 & -- & 32 \\
\hline \multicolumn{12}{|c|}{ Wayne County } \\
\hline East Fork Whitewater River & Whitewater River & 1 & -- & -- & -- & -- & -- & -- & -- & -- & 1 \\
\hline Nolands Fork & Whitewater River & 3 & 3 & -- & -- & -- & 1 & -- & -- & -- & 7 \\
\hline
\end{tabular}




\section{Polyline Shapefile of Investigated Stream Reaches}

A polyline shapefile depicting both active and stationary stream reaches documented in Robinson (2013a), as well as 38 additional tributaries also showing signs of active migration, was derived from line work in the National Hydrography Dataset (NHD), which was downloaded as a statewide extract from The National Map (https://nhd.usgs.gov) on March 15, 2015 (U.S. Geological Survey, 2015). Segmented NHD flow lines were selected between the upstream and downstream points that bound each stream reach and exported from the NHD file geodatabase (.gdb) to shapefile (.shp) format. The names of flow lines in the dataset, sourced from the Geographic Names Information System (U.S. Board on Geographic Names, no date), were edited to be consistent with the names of stream reaches used in Robinson (2013a). After the flow lines were renamed, the dissolve command of ArcInfo was used to combine flow line segments into singlepart features based on contiguity and common names. Lastly, the attributes named main stem, tributary, active, rate_ft_yr, length_mi, and label were added to the attribute table of the shapefile Data_Series_2017_1068_Channel_Migration_Rates. shp (table 4) to support queries on the dataset.

Table 4. Names and descriptions of attributes in Data Series_2017_1068_Channel_Migration_Rates.shp.

\begin{tabular}{|c|c|c|}
\hline Attribute & Description & Data type \\
\hline reach_name & $\begin{array}{l}\text { Name of stream reach, } \\
\text { as documented in } \\
\text { Scientific Investigations } \\
\text { Report 2013-5168 } \\
\text { (Robinson, 2013a) }\end{array}$ & String \\
\hline mainstem & Name of mainstem stream reach & String \\
\hline tributary & $\begin{array}{l}\text { Indicates whether a stream } \\
\text { reach is considered a } \\
\text { mainstem or tributary }\end{array}$ & Short integer \\
\hline active & $\begin{array}{l}\text { Indicates whether a stream } \\
\text { reach is actively migrating } \\
\text { or stationary }\end{array}$ & Short integer \\
\hline rate_ft_yr ${ }^{1}$ & $\begin{array}{l}\text { Channel migration rate } \\
\text { expressed in feet per year }\end{array}$ & String \\
\hline length_mi & $\begin{array}{l}\text { Length of stream reach } \\
\text { expressed in miles along } \\
\text { stream's path }\end{array}$ & Float \\
\hline label & References map label in figure 1 & String \\
\hline
\end{tabular}

${ }^{1}$ Channel migration rates were measured in a report by Robinson (2013a) for mainstem stream reaches only.

\section{Accessing the Data}

The products of this report are available through a data release at https://doi.org/10.5066/F7ZG6R49 (Sperl, 2017). The products include (1) a comma-delimited text file (.csv) of table 3, which provides numbers of vulnerable assets aggregated by stream reach, county, and class of asset; and (2) a polyline shapefile (.shp) of the stream reaches documented in Robinson (2013a) as well as 38 additional tributaries where recent channel migration was observed. Federal Geographic Committee metadata is provided for both files in the associated data release.

\section{References Cited}

Indiana Department of Natural Resources, 2016, Fluvial Erosion Hazard FL, ISJ: variable scale accessed June 1, 2017, at https://indnr.maps.arcgis.com/home/item.html?id=7ba732 3e95e64344b1eb25eff2fb4a9e\#overview.

Indiana Geological Survey, Center for Geospatial Data Analysis, 2002, Natural Gas, Crude Oil, and Refined Oil Pipelines in Indiana: scale 1:63,360, accessed July 1, 2014, at http://maps.indiana.edu/metadata/Infrastructure/Energy_ Pipelines_Oil_Gas.html.

Indiana Silver Jackets, 2017, Official web site of the Indiana Silver Jackets, accessed June 1, 2017, at https://silverjackets.nfrmp.us/State-Teams/Indiana.

Indiana University-Purdue University Indianapolis, 2017, Fluvial Erosion Hazard Program web site, accessed June 1, 2017, at http://feh.iupui.edu/.

Morlock, S.E., Menke, C.D., Arvin, D.V., and Kim, M.H., 2008, Flood of June 7-9, 2008, in central and southern Indiana: U.S. Geological Survey Open-File Report 2008-1322, 15 p., 3 app.

Robinson, B.A., 2013a, Recent (circa 1998 to 2011) channelmigration rates of selected streams in Indiana: U.S. Geological Survey Scientific Investigations Report 2013-5168, 14 p., accessed June 1, 2017, at http://pubs.usgs.gov/ $\operatorname{sir} / 2013 / 5168 /$.

Robinson, B.A., 2013b, Regional bankfull-channel dimensions of non-urban wadeable streams in Indiana: U.S. Geological Survey Scientific Investigations Report 2013-5078, 33 p.

Sperl, B.J., 2017, Associated files for USGS Data Series 2017-1068 on vulnerable transportation and utility assets near actively migrating streams in Indiana: U.S. Geological Survey data release, https://doi.org/10.5066/F7ZG6R49.

U.S. Board on Geographic Names, accessed March 15, 2015, at https://geonames.usgs.gov/domestic/index.html.

U.S. Geological Survey, 2015, The National Map: U.S. Geological Survey web site, accessed March 15, 2015, at https://nhd.usgs.gov. 


\section{Glossary}

ArcInfo GIS software developed by Environmental Systems Research Institute.

Fluvial erosion hazard The suite of risks to structures, property, and infrastructure elements that are brought about by the natural processes of stream-bank erosion and stream-channel meandering.

Fluvial plain The valley-floor (or valley-flat) land that has been produced by fluvial processes and may include the flood plain and adjacent terraces.

Migration rate The rate at which a channel migrates laterally, expressed in units of feet per year.

Stream reach A section of a stream or river between two points.

Main stem A larger river or stream formed by a number of smaller tributaries.

Orthophotography Refers to aerial photographs that have been geometrically corrected to preserve true distances.

Tributary A smaller river or stream that flows into a larger river or stream.

GIS Geographic information system(s).

Google Earth A software application developed by Google with functionality to view imagery from different sources, times, and places. 
For additional information contact:

Director, Indiana Water Science Center U.S. Geological Survey

5957 Lakeside Boulevard

Indianapolis, IN 46278-1996 



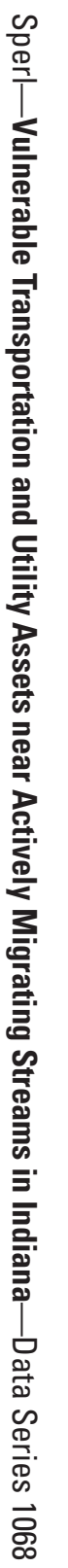

